\title{
Multi-start local search for online scheduling in parallel operating theatre
}

\begin{abstract}
The present work is considered online operating theatre scheduling for regular patients and also it is involving emergency patients on a day. The emergency patients urge to schedule the earliest as their level of priority is higher than the regular patients and it is the importance of online scheduling problem. The local search (LS) method was tested for scheduling the parallel operating theatre with an objective to minimize the delay and overtime cost of operating theatre. A modification of the local search algorithm was performed to improve the solutions and it is called multi-start local search (MSLS). This research found that, MSLS method performed better than LS method since it has reduced the total cost of the operating theatre significantly.
\end{abstract}

Keyword: Health care; Local search; Online scheduling; Scheduling operating theatre 